Hypothesis $3 \mathrm{~T}$ MRI of the carotid artery can identify atherosclerotic plaque rupture in patients presenting with TIA or minor stroke.

Methods 81 patients with carotid artery disease were recruited; 41 presented acutely with TIA or minor stroke and 40 asymptomatic patients acted as the control group. Median time from symptom onset to MRI in the symptomatic group was 2.1 days (range 0.17-7.0). All patients underwent T1, T2 and proton densityweighted turbo spin echo MRI to $10 \mathrm{~mm}$ either side of the carotid. As part of a combined scan protocol, study participants then underwent diffusion-weighted imaging (DWI) and Fluid-Attenuated Inversion Recovery (FLAIR) imaging of the brain to assess acute and chronic injury, respectively. If physically able, patients underwent follow-up scanning a minimum of six weeks later. Plaques were graded according to the MRI modified American Heart Association (AHA) system by two independent reviewers blinded to the clinical status of the patient. Statistical analysis was performed using the Wilcoxon sign rank test and Fisher's exact test to compare plaques, in addition to the Mann Whitney $U$ test to compare cerebral injury. Results AHA type VI (ruptured) plaque was seen in 22/41(54\%) in the symptomatic group vs $8 / 41(20 \%)$ in the asymptomatic group $(p<0.05)$, either due to intra-plaque haemorrhage (34\% vs $18 \%$, $p=0.08$; Abstract 109 figure $1 \mathrm{~A})$, surface rupture ( $24 \%$ vs $5 \%, p=0.03$; Abstract 109 figure $1 \mathrm{~B}$ ), or luminal thrombus (7\% vs $0 \%, p=0.24$; Abstract 109 figure 1C). Of particular note, 17/30 (57\%) cases of AHA VI (ruptured) plaque were seen to cause $<70 \%$ stenosis-the current cut-off for surgical treatment. At follow-up scanning a minimum of 6 weeks later, only two cases of AHA VI plaque showed evidence of full healing. Of the 41 patients in the acute group, evidence of cerebral injury on DWI imaging was seen in 32/41 patients; the median number of lesions per patient was 7 and the median total lesion volume was $10.62 \mathrm{ml}$ (range $0-522 \mathrm{ml}$ ). No significant associations were noted between AHA plaque type and downstream cerebral injury, however the presence of plaque surface rupture independently predicted a higher number of DWI lesions, a higher total DWI burden at presentation, and higher total cerebral FLAIR signal at follow-up when compared to all other plaque types $(p<0.05)$.

A

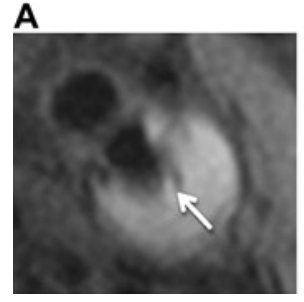

B

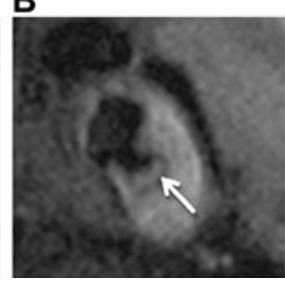

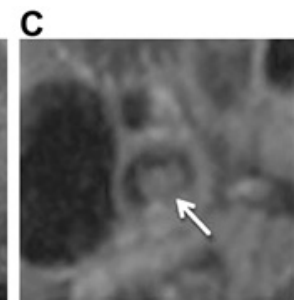

Abstract 109 Figure 1

Conclusion Acute atherosclerotic plaque rupture can be visualised using 3T MRI. In particular, MRI can provide detailed information on plaque morphology that can predict downstream embolic injury, independent of the degree of luminal stenosis caused.

\section{MYOCARDIAL SYSTOLIC STRAIN AND SUBCLINICAL ATHEROSCLEROSIS IN YOUNG ADULT LIFE}

doi:10.1136/heartjnl-2011-300198.110

A J Lewandowski, M Lazdam, E Davis, R Poole, J Diesch, J Francis, D Augustine, R Banerjee, J Suttie, S Neubauer, P Leeson. Cardiovascular Medicine, University of Oxford, Oxford, UK

Background In the elderly, reduced left ventricular function is related to elevated carotid intima media thickness (IMT), a well-established subclinical marker of atherosclerosis. Cardiovascular magnetic resonance (CMR) allows for precise quantification of changes in myocardial structure and function. We therefore sought to determine if in young adults, without overt cardiovascular risk factors, there was already evidence of early changes in systolic function related to subclinical atherosclerosis.

Methods We studied 81 individuals (44 females, 37 males) without cardiovascular risk factors and with a mean age of $28.42 \pm 5.36$ years (mean \pm SD). Peak mid-ventricular myocardial circumferential systolic strain and left ventricular mass adjusted for body surface area (LVM) were assessed by CMR. Carotid IMT was measured as a marker of subclinical atherosclerosis using ultrasound. Demographic and anthropometric characteristics were measured as well as metabolic parameters and peripheral and central blood pressure.

Results Individuals with reduced peak myocardial circumferential systolic strain had higher carotid IMT $(r=0.392, p<0.001)$. Total cholesterol level and waist to hip ratio were both significantly associated with reduced myocardial strain. Increased LVM, central and peripheral systolic blood pressure, peripheral pulse pressure, glucose, triglycerides, age, body mass index and waist to hip ratio, as well as reduced high-density lipoprotein, were all significantly associated with increased carotid IMT $(p<0.01)$. Males also had higher carotid IMT than females (mean $\pm S D=0.54 \pm 0.068 \mathrm{~mm}$ vs $0.47 \pm 0.042 \mathrm{~mm}$, $\mathrm{p}<0.001$ ). The association between carotid IMT and peak myocardial circumferential systolic strain was independent of gender, smoking, LVM as well as peripheral and central blood pressure measures.

Conclusions We have shown for the first time that subclinical changes in cardiac function and subclinical atherosclerosis are closely interrelated in young adults, with associations that extend to those in the normal range of cardiovascular risk. This study further establishes the ability of CMR to detect early changes in cardiovascular disease development.

\section{SINGLE CENTRE PROSPECTIVE CARDIAC CT STUDY TO DETERMINE THE PREVALENCE OF PATIENTS WITH CORONARY ARTERY DISEASE WITH A ZERO CORONARY ARTERY CALCIUM SCORE AND ASSOCIATED NON-CARDIAC INCIDENTAL FINDINGS}

doi:10.1136/heartjnl-2011-300198.111

A J Shah, D R Obaid, D Gopalan, J Babar, J H F Rudd. Addenbrooke's Hospital, Cambridge, UK

Introduction Cardiac CT, incorporating coronary artery calcium (CAC) scoring and angiography, is being increasingly used to evaluate patients with chest pain and exclude coronary artery disease (CAD), as recommended in the recent NICE guidelines. Calcification of the coronary arteries is an excellent marker of underlying atherosclerosis, but a zero CAC score does not completely exclude the diagnosis as potentially significant non-calcified plaques will not be detected by CAC scoring. CT imaging may also identify non-cardiac incidental findings that can lead to further downstream testing and medical expense.

Objectives (1) To evaluate the probability of $\mathrm{CAD}$ in patients with a CAC score of zero. (2) To determine the incidence of non-cardiac incidental findings on cardiac CT and to quantify resulting investigations. Methods 116 symptomatic patients undergoing cardiac CT to exclude CAD from November 2009 to October 2010 were prospectively enrolled. Patients underwent CAC scoring and had contrastenhanced, 128-slice, dual source CT coronary angiography (CTCASiemens Flash). Scans were dual-reported by a cardiac radiologist and a cardiologist. Statistical analysis was performed using GraphPadPrism. Results $62 / 116$ patients had a CAC score of zero. Of these, $57(91.9 \%)$ patients had normal coronary arteries, 4 (6.5\%) patients had nonobstructive $\mathrm{CAD}$ (stenosis $<50 \%$ ), and 1 patient $(1.6 \%)$ had significant obstructive CAD (stenosis $>50 \%$ ). This patient with obstructive $\mathrm{CAD}$ had a high grade lesion in the proximal left anterior descending artery that required intervention. 54/116 had non-zero CAC scores. Of these, 13 (24\%) had obstructive $\mathrm{CAD}$ and 41 (76\%) nonobstructive CAD. 42/116 (36\%) patients had incidental findings on 
cardiac CT that are summarised in Abstract 111 table 1. These incidental findings resulted in further investigations, documented in Abstract 111 table 2. The mean radiation dose ( \pm SEM) for CAC scoring was $0.61 \pm 0.03 \mathrm{mSv}$. The mean radiation dose ( \pm SEM) for subsequent CTCA was $2.66 \pm 0.32 \mathrm{mSv}$ in high pitch "flash" mode $(\mathrm{n}=27), \quad 5.86 \pm 0.50 \mathrm{mSv}$ in prospective mode $(\mathrm{n}=64)$ and $17.15 \pm 1.68 \mathrm{mSv}$ in the retrospective mode $(\mathrm{n}=25)$.

Abstract 111 Table 1 Incidental findings on cardiac CT

\begin{tabular}{lllr}
\hline Area & Structure & Incidental Finding & $\mathbf{n}$ \\
\hline Chest $(\mathrm{n}=27)$ & Lung parenchyma & Nodule $<1 \mathrm{~cm}$ & 5 \\
& & Emphysema & 3 \\
& & Atelectasis & 6 \\
& & Fibrosis & 4 \\
& & Tumour recurrence & 1 \\
& Pleura & Bronchiectasis & 2 \\
& & Effusion & 2 \\
Abdomen $(\mathrm{n}=7)$ & Calcification & 2 \\
Diaphragm $(\mathrm{n}=5)$ & Lymph node & Adenopathy & 2 \\
Vasculature $(\mathrm{n}=11)$ & Adernal & Cyst/Nodules & 6 \\
& & Adenoma/metastasis & 1 \\
& Aorta & Hiatus Hernia & 5 \\
& & Dilatation & 8 \\
& Renal & Aneurysm & 1 \\
& Coeliac & Stenosis & 1 \\
& & Stenosis & 1 \\
\hline
\end{tabular}

Abstract 111 Table 2 Further investigation of incidental findings on Cardiac CT

\begin{tabular}{lr}
\hline Investigation & $\mathbf{n}$ \\
\hline Bone scintigraphy & 1 \\
Chest clinic referral & 2 \\
CT chest & 4 \\
DMSA & 1 \\
MR adrenals & 1 \\
MRA renal & 1 \\
Nephrology clinic referral & 1 \\
Pleural fluid aspiration & 1 \\
Ultrasound kidneys & 1 \\
Ultrasound liver & 3 \\
\hline
\end{tabular}

Abstract 111 Table 3 Investigations and referrals generated by incidental findings

\begin{tabular}{lc}
\hline Investigations or referrals & Number \\
\hline Bone scintigraphy & 1 \\
Chest clinic referral & 2 \\
CT chest & 4 \\
DMSA & 1 \\
MR adrenals & 1 \\
MR cardiac & 2 \\
MRA renal & 1 \\
Nephrology clinic referral & 1 \\
Pleural fluid aspiration & 1 \\
Ultrasound kidneys & 1 \\
Ultrasound liver & 3
\end{tabular}

Conclusions Despite 62 patients having a reassuring CAC score of zero, $8 \%$ of this group had evidence of non-calcified plaque, with one patient having obstructive $\mathrm{CAD}$ that required intervention. We conclude that if strong clinical suspicion remains in patients with a CAC score of zero further coronary investigation may be warranted. Incidental findings are common, and can result in multiple further investigations for patients. Further research is needed to evaluate the added cost, clinical benefits and radiation exposure created by investigation of such incidental findings in the context of cardiac CT.

\section{COMPUTED TOMOGRAPHIC CORONARY ANGIOGRAPHY TO SCREEN FOR ALLOGRAFT VASCULOPATHY AFTER HEART TRANSPLANTATION}

doi:10.1136/heartjnl-2011-300198.112

M G Panicker, A G Mitchell, N R Banner, T K Mittal. Harefield Hospital, Harefield, UK

Objective To evaluate ComputedTomographic Coronary Angiography (CTA) as an alternative to Invasive Coronary Angiography (ICA) for the detection of Cardiac Allograft Vasculopathy (CAV).

Background CAV is an important cause of late mortality after heart transplantation (HT). Because patients are often asymptomatic, surveillance ICA is performed in our institution. CTA is effective for the diagnosis of coronary disease in non-transplant patients, but few studies have been done after HT.

Methods $117 \mathrm{HT}$ patients, 1 to 24 years post transplant (mean=12 years SD \pm 6 ) underwent CT coronary artery calcification (CTCAC) followed by retrospective ECG gated coronary angiogram on a 64slice scanner without the use of any $\beta$-blockers. Majority (89\%) of patients had CTA within $24 \mathrm{~h}$ before ICA. The Agatston calcium score (CS) was calculated for all patients. The CTA images were systematically analysed for image quality and the presence of CAV (graded as significant if $>50 \%$ luminal stenosis) using a fifteen coronary segments model by an independent investigator blinded to the results of ICA.

Results CS ranged from 0 to 1681 (Mean=91.7 \pm 275 ). Out of 77 patients with absent CS, 3 had significant CAV on ICA. Despite a mean resting heart rate of $82 \mathrm{bpm} S D \pm 13$ and body mass index of $27 \mathrm{~kg} / \mathrm{m}^{2} \mathrm{SD} \pm 5,81 \%$ of the CTA images were graded as excellent or satisfactory. For all the 1755 segments assessed by CTA irrespective of the image quality, CTA had sensitivity, specificity, positive and negative predictive values of $71 \%, 79 \%, 72 \%$ and $78 \%$ respectively for the detection of any CAV found by ICA. On a patient basis, CTA best performed in diagnosing CAV of more than $25 \%$ with sensitivity, specificity, positive and negative predictive values of $74 \%, 94 \%, 79 \%$, and $92 \%$ respectively. None of the 61 patients with completely normal CTA had CAV on ICA. $83(92 \%)$ out of 90 patients who responded to a patient survey preferred CTA to ICA as a screening test for CAV. Non-coronary cardiac and non-cardiac abnormalities were identified in $18 \%$ and $14 \%$ patients respectively.

Conclusion The study shows that CTA compares favourably with ICA in detecting CAV in heart transplant recipients, and may be a preferable screening technique because of its non-invasive nature, patient preference and yield of additional information. One has to exercise caution in just using CS in these patients as significant CAV can be missed out.

\section{DUAL ENERGY CT IMPROVES DIFFERENTIATION OF CORONARY ATHEROSCLEROTIC PLAQUE COMPONENTS COMPARED TO CONVENTIONAL SINGLE ENERGY CT}

doi:10.1136/heartjnl-2011-300198.113

${ }^{1} \mathrm{D}$ R Obaid, ${ }^{1} \mathrm{P}$ A Calvert, ${ }^{1} \mathrm{~J} \mathrm{H}$ F Rudd, ${ }^{2} \mathrm{D}$ Gopalan, ${ }^{1} \mathrm{M}$ R Bennett. ${ }^{1}$ University of Cambridge, Cambridge, UK; ${ }^{2}$ Papworth Hospital NHS Trust, Cambridge, UK

Introduction Vulnerable plaques have a relatively high necrotic core area and low fibrous tissue content. Although CT can identify plaque components on the basis of their x-ray attenuation, there is 